Section: Bioprocessing and Biological Engineering

\title{
Simple control of fed-batch processes for recombinant protein production with $E$. coli
}

Sebastian Schaepe* sebastian.schaepe@biochemtech.uni-halle.de

Artur Kuprijanov*» artur.kuprijanov@biochemtech.uni-halle.de

Mathias Aehle* mathias.aehle@biochemtech.uni-halle.de

Rimvydas Simutis ${ }^{\S}$ rimvydas.simutis@ktu.lt

Andreas Lübbert* andreas.luebbert@biochemtech.uni-halle.de (corresponding author)

Phone: 03455525942

Fax: 03455527288

* Institute of Biochemistry and Biotechnology, Martin-Luther-University Halle-Wittenberg, Kurt-Mothes-Straße 3, 06120 Halle(Saale), Germany

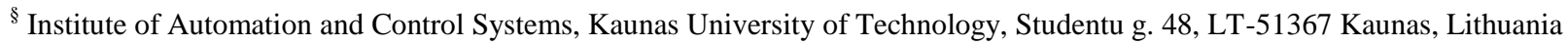




\begin{abstract}
A very simple but effective process control technique is proposed that leads to a high batch-to-batch reproducibility with respect to biomass concentration as well as the specific biomass growth rate profiles in E.coli fermentations performed during recombinant protein production. It makes use of the well-established temperature controllers in currently used fermenters, but takes its information from the difference between the controlled culture temperature $\mathrm{T}_{\text {cult }}$ and the temperature $\mathrm{T}_{\text {coolin }}$ of the coolant fed to the fermenter's cooling jacket as adjusted by the fermenter temperature controller. For process control purposes this measured difference is corrected regarding stirrer influences and cumulated before it is used as a new process control variable. As a spin-off of this control, it becomes possible to estimate online the oxygen mass transfer rates and the corresponding $\mathrm{k}_{\mathrm{L}}$ a values during the real cultivation process.
\end{abstract}

Keywords: Fed-Batch, Heat flow, High cell density cultivation, Protein production

\title{
Introduction
}

We have shown that cultures of E.coli producing recombinant proteins can be controlled to very high batch-to-batch reproducibility using the profiles of the total amount of $\mathrm{CO}_{2}$ produced by the cells (Jenzsch et al. 2007, Gnoth et al. 2007, 2010). When such control techniques are applied at industrial production reactors the question appears how to precede when the off-gas analyzers fail. In that case, it is possible to immediately switch to open-loop control or to reconstruct the total cumulated $\mathrm{CO}_{2}$ production rate (tcCPR) measurement values from other online variables. Here we present another simple alternative: namely to switch to another simple controller, the control variable of which can be measured at all production reactors, i.e. the temperature difference between the coolant fed to the cooling jacket and the culture temperature. For a given reactor, such a controller can be tuned in such a way that the control quality with respect to the tcCPR-controller will not significantly be reduced.

The control technique makes use of the temperature controller installed at every production fermenter. As it generally keeps the culture temperature $\mathrm{T}_{\text {cult }}$ constant, the information about the changes in the heat generation during the fermentation process must be derived from its actions. The main action of most temperature controllers is adjusting the temperature of the coolant supplied to the fermenter's heat exchanger, e.g. the cooling jacket, in such a way that $\mathrm{T}_{\text {cult }}$ is kept on its desired value. From the coolant flow rate and its temperature difference, the heat transferred to the fermenter can be estimated. When additional estimates about the power input to the culture by means of the agitator are available, then the metabolic heat can be estimated. The power input by the agitator is usually derived from correlations 
established from experiments in model media. Here we show that these engineering parameters can also be derived from measurements performed during real cultivations.

\section{Materials and methods}

E.coli BL21(DE3) pLysS was used in all experiments. Cells were inoculated in a defined medium. From the beginning, i.e. starting with the inoculation, all cultivations were run in the fed-batch mode. Initial medium and feed solution are described in detail by (Gnoth et al. 2010).

All cultivations were performed in a Biostat C10 fermenter (B.Braun Biotech International, Melsungen, Germany) with diameter $\mathrm{T}=0.185 \mathrm{~m}$ run under the control of B.Braun Biotech International's Digital Control Unit (DCU). The fermenter was equipped with three home-made hollow blade impellers with diameters $\mathrm{D}=0.5 \mathrm{~T}$. Four enlarged baffles of $30 \mathrm{~mm}$ width were installed within the fermenter. Except for the initial phase, the aeration was constant at $4 \mathrm{vvm}$. A temperature sensor (Wika, TR-30W Pt100 B2) was installed at the entrance of the cooling jacket of the fermenter. The

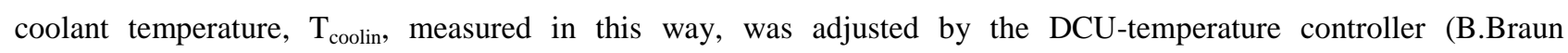
International) in such a way that the culture temperature was kept exactly at $\mathrm{T}_{\text {cult }}=35{ }^{\circ} \mathrm{C}$. The mass flow rate through the jacket was taken from the data-sheet of the coolant circulation pump (Grundfos UP32-20N) of the fermenter and kept on a fixed value of $0.3 \mathrm{~kg} / \mathrm{s}$. The flow rate value was confirmed in a stimulus response experiment. By measuring the time between two short heating periods, we calculated the mass flow through the jacket (jacket volume was 1.81 ).

The $\mathrm{pH}$ in the culture was precisely controlled to 7 by addition of $25 \% \mathrm{NH}_{4} \mathrm{OH}$.

\section{Temperature-based process controller}

The controller developed in this work is a very simple feedforward/feedback controller of the PID-type. It measures the difference $\Delta \mathrm{T}$ between the temperature $\mathrm{T}_{\text {cult }}$ in the culture and the temperature $\mathrm{T}_{\text {coolin }}$ of the coolant fed to the cooling jacket and uses the substrate feed rate as the control variable. As $\mathrm{T}_{\text {cult }}$ is controlled to stepwise fixed values in the fermentation system, the information about the heat development in the culture can be estimated from the temperature variations in the cooling water fed to the heat exchanger by the DCU temperature controller.

In real cultivation processes, heat is also generated by the stirrer. Consequently, for reliable process control the difference $\Delta \mathrm{T}$ must be corrected by a special estimation procedure that eliminates the influence of stirrer action on the cultivation temperature. These corrected temperature difference profiles $\Delta \mathrm{T}_{\mathrm{c}}(\mathrm{t})$ could directly be used as set-point 
profiles for the controller. However, in order to avoid influence of short time disturbances (feed pump disturbances, antifoam influence) on the bioprocess quality, it is of advantage to use cumulated $\Delta T_{c}(t)$ profiles cum $\Delta T_{c}(t)$ as set point profiles for the controller. This strategy is in a modified version also applicable for open cooling systems, were the coolant flow rate is regulated.

The control variable of the controller is the substrate solution feed rate $\mathrm{F}(\mathrm{t})$, which is fed to the culture. The controller takes the difference between the real value of $\operatorname{cum} \Delta \mathrm{T}_{\mathrm{c}}(\mathrm{t})$ and corresponding value of the setpoint profile cum $\Delta \mathrm{T}_{\mathrm{c}}(\mathrm{t})_{\text {set }}$ and uses the following simple control law to estimate the substrate feed rate $\mathrm{F}(\mathrm{t})$.

$F(t)=F_{r e f}(t) \cdot(1+u(t)) u(t)=K_{c}\left(e(t)+\frac{T_{c y c}}{T_{i}} \cdot \sum_{t=0}^{t} e(t)+\frac{T_{d}}{T_{c y c}} \cdot(e(t)-e(t-1))\right)$

$e(t)=\operatorname{cum} \Delta T_{c}(t)_{s e t}-\operatorname{cum} \Delta T_{c}(t)$

where $F_{\text {ref }}(t)$ is the feed-forward part of the controller implemented as a reference feed profile, $e(t)$ is the deviation from the setpoint, $K_{c}$ is the control gain, $T_{i}$ and $T_{d}$ are the parameters of the integral and derivative terms, $T_{\text {cyc }}$ is the control cycle. In the cultivation experiments we initially used following set of PID controller parameters obtained with the tuning procedure of Ziegler and Nichols: $K_{c}=95, T_{i}=150 \mathrm{~s}, T_{d}=30 \mathrm{~s}$, and $\mathrm{T}_{\text {cyc }}=10 \mathrm{~s}$.

The entire control system, based on measurements of the temperature of the coolant fed to the cooling jacket and temperature in the bioreactor is sketched in Fig 1.

\section{Fig lhere}

The feedforward component of the controller directly uses the substrate feed reference profile $F_{\text {ref }}$ This already considers the variations in the dynamics of the culture as faster growing cultures need higher feed rates to keep the physiological state of the cells as characterized by their specific growth rate $\mu$ at the desired level.

The reference profile $\mathrm{F}_{\text {ref }}$, and $\operatorname{cum} \Delta \mathrm{T}_{\mathrm{c}}(\mathrm{t})_{\text {set }}$ can be taken from well-established, previously performed tcCPR-controlled cultivations. This makes this controller so simple to construct and to use as a backup controller of tcCPR controlled 
cultures. If there are no tcCPR feed reference profiles available, historical fermentation data with the best cultivation results can be used instead. As the heat generation in the culture as well as the heat transfer rates depend of the stirrer speed, this influence was analyzed in a separate experiment. Fig 2 shows the temperature changes upon changes in the stirrer speed. As can be seen, while the culture temperature is still under control, the coolant temperature changes with the impeller speed in a slightly nonlinear way. Of course, the change is specific for a given reactor and given culture.

Fig 2here

The correlation between stirrer speed and resulting jacket temperature could be fitted to a simple second order polynomial.

$T_{j}=(9.91 \mathrm{e}-7 \pm 0.051 \mathrm{e}-7) \cdot$ Stirr $^{2}+(6.12 \mathrm{e}-5 \pm 0.578 \mathrm{e}-5) \cdot$ Stirr $+35.42 \pm 0.02$

This simple equation was then used to compute the corrected $\Delta \mathrm{T}_{\mathrm{c}}(\mathrm{t})_{\text {set }}$, and the set-profile cum $\Delta \mathrm{T}_{\mathrm{c}}(\mathrm{t})$ for the control system.

$$
\Delta T_{c}(t, \text { Stirr })=\left(T_{\text {cult }}(t)-T_{\text {coolin }}(t)\right)-\left(T_{\text {cult }}(t)-T_{j}(\text { Stirr })\right)
$$

\section{Fig 3here}

This information about $\Delta \mathrm{T}_{\mathrm{c}}(\mathrm{t})$ can also be used for indirect estimates of the carbon dioxide production rate. The relationship between $\Delta \mathrm{T}_{\mathrm{c}}(\mathrm{t})$ and the total carbon dioxide production rate tCPR appears to be linear.

$t C P R=(46.59 \pm 0.13) \cdot \Delta T_{c}+12.57 \pm 0.08$

This relation was used in the lower part of Fig 3 to estimate the tCPR profiles for different fermentations, which are known to contain the key information about the dynamics of the cultivation process.

\section{Results}

\section{Direct control}

Reference for this temperature-based control procedure is the cumulated difference between the culture temperature and the temperature of the coolant entering the cooling jacket adjusted for stirrer influence during our cultivation run with 
the database identifier S706. This cultivation and a couple of subsequently controlled cultivations based on this desired $\operatorname{cum} \Delta \mathrm{T}_{\mathrm{c}}(\mathrm{t})$ profile are shown in Fig 4.

The controller was started at fermentation time $\mathrm{t}=8 \mathrm{~h}$ since the signal-to-noise ratio of the temperature signal at lower temperatures was considered too low by the same argumentation as applied to the tcCPR controller (Jenzsch et al. 2007). The control error may be large at the beginning of the controlled phase, but it becomes very small after some cultivation time.

\section{Fig 4here}

$\Delta \mathrm{T}_{\mathrm{c}}(\mathrm{t})$ profile for these cultivation runs are shown in Fig 5. In these data, the heat generated by the impeller system is already eliminated. When sufficiently high substrate conversion rates appear, i.e. at fermentation times $\mathrm{t}>8 \mathrm{~h}$, the temperature difference profile $\Delta \mathrm{T}_{\mathrm{c}}(\mathrm{t})$ resembles the $\mathrm{O}_{2}$ uptake rate and thus also the $\mathrm{CO}_{2}$ production rate to a sufficient accuracy.

Fig 5here

\section{Indirect control of the biomass concentration}

As one is not primarily interested in controlling temperature differences $\Delta \mathrm{T}_{\mathrm{c}}(\mathrm{t})$, it is straightforward to look for the key variables of the process, namely the specific growth rate $\mu$ and biomass concentration $\mathrm{X}$.

The biomass concentration profiles of the $\operatorname{cum} \Delta \mathrm{T}_{\mathrm{c}}(\mathrm{t})$-controlled cultivations are depicted in Fig 6. They are estimated using a full dynamic process model from the measured values of the online variables [t, W, F, OUR, CPR] which are available under normal process conditions. As can be seen in the figure, the batch-to-batch reproducibility with respect to biomass is perfect using this control technology. Nearly the same performance as with the tcCPR control was obtained. Hence this temperature difference controller can be used as stand-by controller in this kind of cultivations.

\section{Fig 6 here}

$\sigma_{\max }=1.93 \pm 0.061 / \mathrm{h}$

$K_{S}=0.073 \pm 0.02 \mathrm{~g} / 1$

$Y_{X S}=0.38 \pm 0.01 \mathrm{gX} / \mathrm{gS}$ As the profile of the reference cultivation $\mathrm{S} 706$ was taken from a fermentation which was $K_{X}=167.2 \pm 9.8 \mathrm{gX} / \mathrm{kg}$

$n=0.69 \pm 0.07$

designed to run a certain trajectory of the specific growth rate, it is interesting to have a view on the specific growth rate estimates $\mu(t)$ for the $\operatorname{cum} \Delta \mathrm{T}_{\mathrm{c}}(\mathrm{t})$-controlled fermentations. These are shown in Fig 7 . We see that not only the biomass 
concentrations but even the $\mu$ profiles, estimated with the same full dynamic process model, are exactly reproduced in the plot. This says that the controller performance is quite high and the deterministic growth process is well described by the process model.

$$
\begin{aligned}
& \sigma=\frac{\sigma_{\max } \cdot S}{K_{S}+S} \\
& \mu=\sigma \cdot Y_{X S} \cdot\left(1-\frac{X}{K_{X}-X}\right)^{n}
\end{aligned}
$$

The parameters are calculated by means of the Nelder-Mead simplex optimization approach implemented in Matlab.

\section{Fig 7 here}

The results depicted in Fig 6 and Fig 7essentially show that the proposed control technique does not only keep the process on the desired path of the controlled variable but that all other state variables are kept on track as well. We are consequently permitted to assume that the oxygen uptake rate profile is in accordance to the biomass growth profile. This allows the estimation of some engineering parameters that will be discussed next.

\section{Discussion}

\section{Reactor engineering aspects}

The complete general energy balance around the culture depends on several terms. The only significant contributions to the temperature control application described here are $\mathrm{Q}_{\mathrm{met}}$, the rate of metabolic heat generation and $\mathrm{Q}_{\mathrm{ag}}$, the rate of heat generated by agitation.

The rate of metabolic heat generation $\mathrm{Q}_{\mathrm{met}}$ can be estimated using the measured $\mathrm{O}_{2}$ uptake rate OUR $\left[\mathrm{molO}_{2} \mathrm{~m}^{-3} \mathrm{~s}^{-1}\right]$.

$$
\mathrm{Q}_{\mathrm{met}}=\mathrm{Y}_{\mathrm{ho}} \mathrm{q}_{\mathrm{o}} \mathrm{XV}=\mathrm{Y}_{\mathrm{ho}} \mathrm{OUR} \mathrm{V}
$$

where $\mathrm{Y}_{\mathrm{ho}}=460\left[\mathrm{~kJ}\left(\mathrm{molO}_{2}\right)^{-1}\right]$ is the heat per oxygen yield, $\left.\mathrm{q}_{0}[\mathrm{~mol} \mathrm{O} \text { ( } \mathrm{g} \text { biomass })^{-1} \mathrm{~m}^{-3} \mathrm{~s}^{-1}\right]$ is the specific rate of $\mathrm{O}_{2}$ consumption. $\mathrm{V}\left[\mathrm{m}^{3}\right]$ is the volume of the culture which was taken from the measured culture weight $\mathrm{W}$.

On the other hand, the heat removed from the reactor can be estimated from the temperature difference at the cooler and the coolant flow rate. The results for the total heat removal and the metabolic heat are compiled in Fig 8 . As can be 
seen, in laboratory-scale fermenters, extremely high mechanical heat production rates appear compared to the metabolic heat production rates.

Compared to these two factors, the other rates of heat generation and loss can be neglected. Even in cultures with smaller growth rates, the contributions to heat generation by stirring and metabolism are dominant (e.g., Biener et al. 2010).

\section{Fig 8 here}

The differences between the total heat production and the metabolic heat generation can thus be attributed to the power transferred into the culture by the agitator. Using the online recorded culture weights, data of the specific power inputs to the culture can be determined. These are shown in Fig 9.

\section{Fig 9 here}

Note that the maximal specific power input is about $0.3 \mathrm{~kW} / \mathrm{kg}$. This is, of course, much higher than the values that are possible in production reactors, but explains the very high $\mathrm{k}_{\mathrm{L}} \mathrm{a}$ values that were obtained in these cultures. $\mathrm{k}_{\mathrm{L}} \mathrm{a}$ values up to more than $3000\left[\mathrm{~h}^{-1}\right]$ were recorded as can be seen in Fig 10. They are in agreement with estimates based on the biomass formed during the cultivation process.

Fig 10 here

Then, consequently, it is possible to make use of a relationship between $\mathrm{k}_{\mathrm{L}} \mathrm{a}$ and the corresponding specific power inputs $\mathrm{P} / \mathrm{W}$ to the culture by the stirrer. This is shown in the correlation plot depicted in Fig 11.

\section{Fig 11 here}

$k_{L} a=\alpha\left(\frac{P}{W}\right)^{\beta}$ For that part of the curve for which the aeration rate was kept constant, we can fit the model most often applied in literature (e.g., Van't Riet 1979) to these data.

The fit to the data in Fig 11 yielded the parameter values

$\alpha=2.79 \pm 0.09$

$\beta=0.72 \pm 0.007$ 
This provides a possibility to compare the result with the correlations shown in literature, which are mainly developed for air in water dispersions.

\section{Conclusions}

1. The controller uses very simple measurement devices and proved to be a perfect backup controller for fed batch processes controlled with tcCPR control.

2. The controller perfectly controls the process indirectly with respect to its specific growth rate and its biomass profiles.

3. If there is no offgas analyzer implemented, the controller can also be used as a standalone controller.

4. Unmeasured heat losses are not influencing the control performance of the analyzed bioreactor.

5. In a regularly working process with offgas analysis, the measurement variables can be used to estimate realistic values of the $\mathrm{k}_{\mathrm{L}}$ a vs. powerdraw relationships. Such results are very scarce for real fermentation environments.

\section{Acknowledgements}

The financial support from the Ministry of Science and Education by means of the Excellence-Initiative Sachsen-Anhalt is gratefully acknowledged. We also want to thank the Research Council of Lithuania for their financial support.

\section{References}

Biener R, Steinkamper A, Hofmann J (2010) Calorimetric control for high cell density cultivation of a recombinant Escherichia coli strain. J Biotechnol 146:45-53

Gnoth S, Jenzsch M, Simutis R, Lübbert A (2007) Process Analytical Technology (PAT): Batch-to-batchreproducibility of fermentation processes by robust process operational design and control. J Biotechnol 132:180-186 Gnoth S, Simutis R, Lübbert A (2010) Selective expression of the soluble product fraction in Escherichia coli cultures employed in recombinant protein production processes. Appl Microbiol Biotechnol 87:2047-2058

Jenzsch M, Gnoth S, Kleinschmidt M, Simutis R, Lübbert A (2007) Improving the batch-to-batch reproducibility of microbial cultures during recombinant protein production by regulation of the total carbon dioxide production. $\mathrm{J}$ Biotechnol 128:858-867 
Meier-Schneiders M, Grosshans U, Busch C, Eigenberger G (1992) Investigation and control of fermentation processes using fermenter calorimetry. 403-407 in: Bioprocess engineering, monitoring and controlling, applied genetics and safety, low molecular weight metabolites, environmental biotechnology, Kreysa G, ed., Dechema Biotechnology Conferences, 5, VCH, Weinheim

Roels J A (1983) Energetics and kinetics in biotechnology. Elsevier Biomedical Press, Amsterdam Van’t Riet K (1979) Review of Measuring Methods and Results in Nonviscous Gas-Liquid Mass Transfer in Stirred Vessels. Ind Eng Chem Proc Des Dev 18:357-364

Yang J-D, Wang N S (1992) Oxygen mass transfer enhancement via fermentor headspace pressurization. Biotechnol Prog 8:244-251

\section{Figure Captions}

Fig 1: Control scheme for the fed-batch cultivation process

Fig 2: Heat production by stirring. A: The system calibration was performed in the liquid medium without cells. Reactor weight, $\mathrm{W}=5 \mathrm{~kg}$, aeration rate was controlled at $4 \mathrm{vvm}$. B: Correlation plot with polynomial estimation of the jacket temperature based on data showed in part $\mathrm{A}$

\begin{tabular}{|l|}
\hline Jacket Temperature \\
Estimation of Jacket Temperature \\
Reactor Temperature \\
Stirrer Speed
\end{tabular}

Fig 3: A: linear correlation between $\Delta \mathrm{T}_{\mathrm{c}}(\mathrm{t})$ and total carbon dioxide production rate. $\mathrm{B}$ : total carbon dioxide production rate of the culture as derived from the temperature profiles measured as compared with tCPR computed from the offgas volume fractions.

Fig 4: A: Patterns of the control variable recorded in four fermentation runs, together with the setpoint profile $\operatorname{cum} \Delta \mathrm{T}_{\mathrm{c}}(\mathrm{t}) . \mathrm{B}$ : Corresponding control errors for these cultivations.

Fig 5: Measured temperature $\Delta \mathrm{T}_{\mathrm{c}}(\mathrm{t})$ at the entrance of the jacket cooler as a function of time. Note, the temperature within the culture is tightly controlled to $\mathrm{T}=35^{\circ} \mathrm{C}$. The substrate feed profile of cultivation S706 was used for the determination of reference profile $\mathrm{F}_{\text {set }}(\mathrm{t})$. 
Fig 6: Profiles of the biomass concentration values estimated for the various $\operatorname{cum} \Delta \mathrm{T}_{\mathrm{c}}(\mathrm{t})$ controlled cultivations.

Fig 7: Profiles of the specific biomass growth rates estimated for the various $\Delta \mathrm{T}_{\mathrm{c}}$ controlled cultivations corresponding to Fig 6 .

Fig 8: Heat removal rate $\mathrm{Q}_{\mathrm{g}}$ and metabolic heat production rate $\mathrm{Q}_{\mathrm{m}}$ as a function of the cultivation time for fermentation S662.

Fig 9: Specific power input to the fermenter as a function of time.

Fig 10: $\mathrm{k}_{\mathrm{L}} \mathrm{a}$ values estimated from the oxygen uptake rates and the measured dissolved oxygen concentrations. The saturation concentration of oxygen $\mathrm{O}^{*}$ was calculated by a logarithmic mean of the inlet and outlet volume fractions of oxygen.

Fig 11: Oxygen transfer coefficient $\mathrm{k}_{\mathrm{L}} \mathrm{a}$ as a function of the specific power input measured during the entire fermentation run S662.

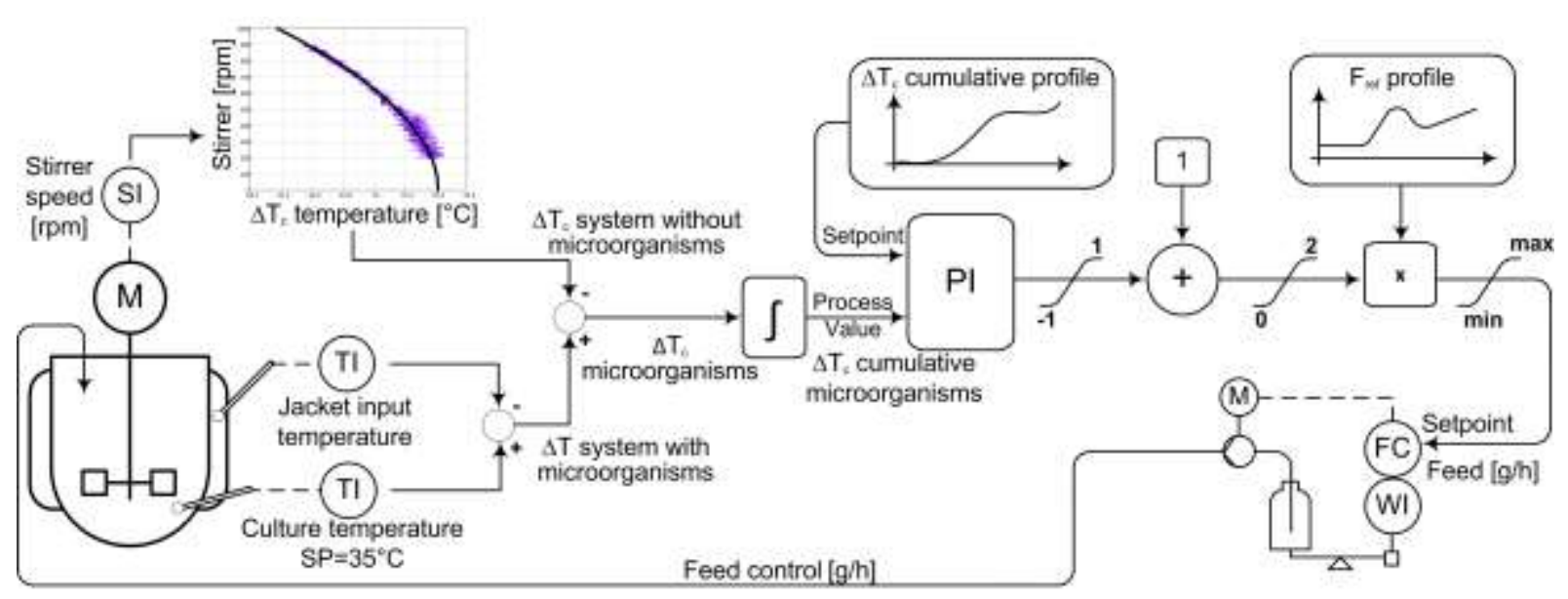



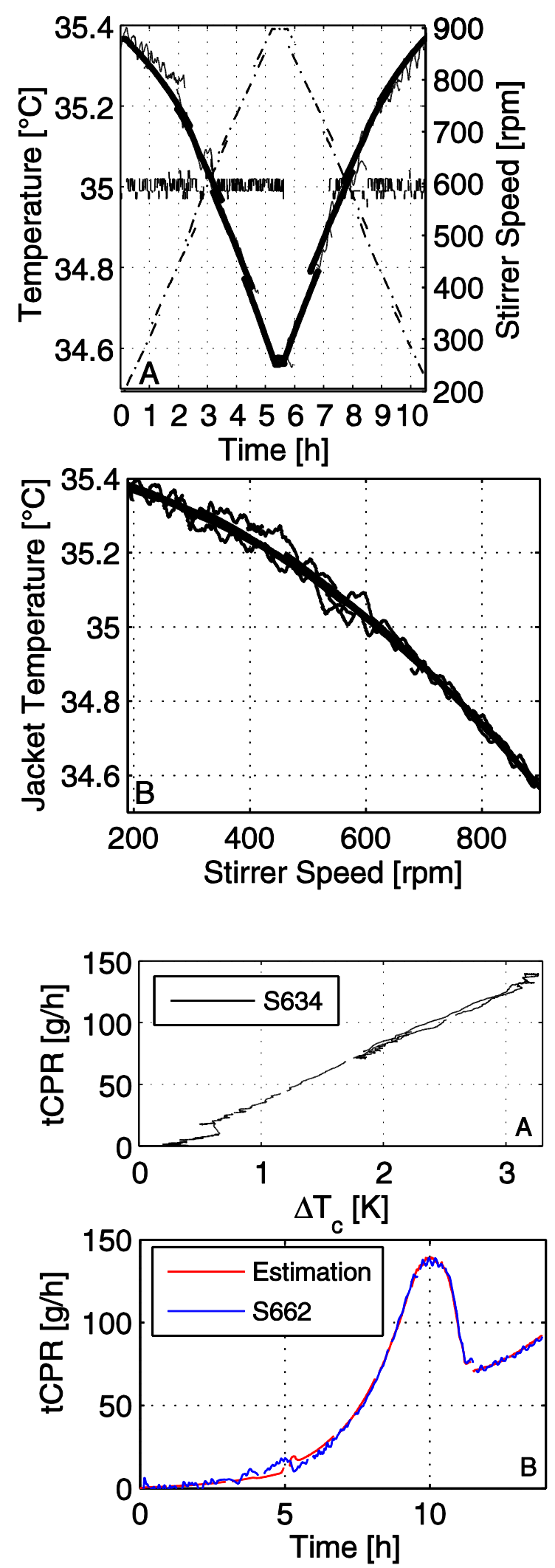

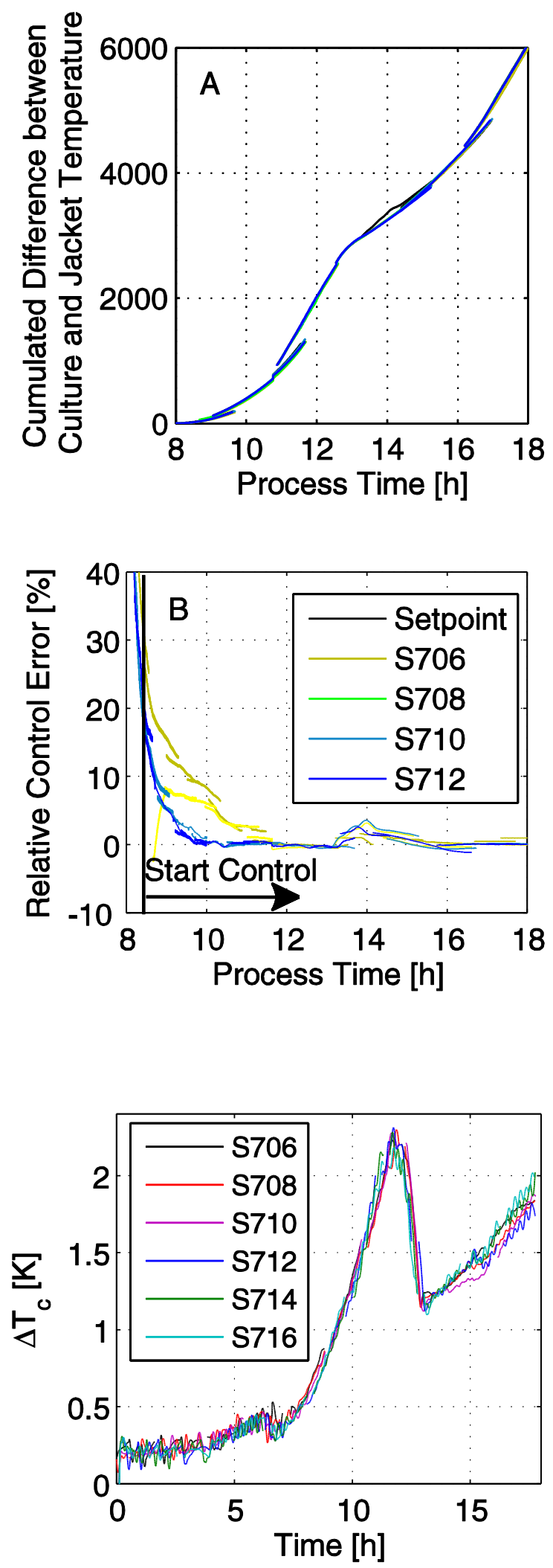

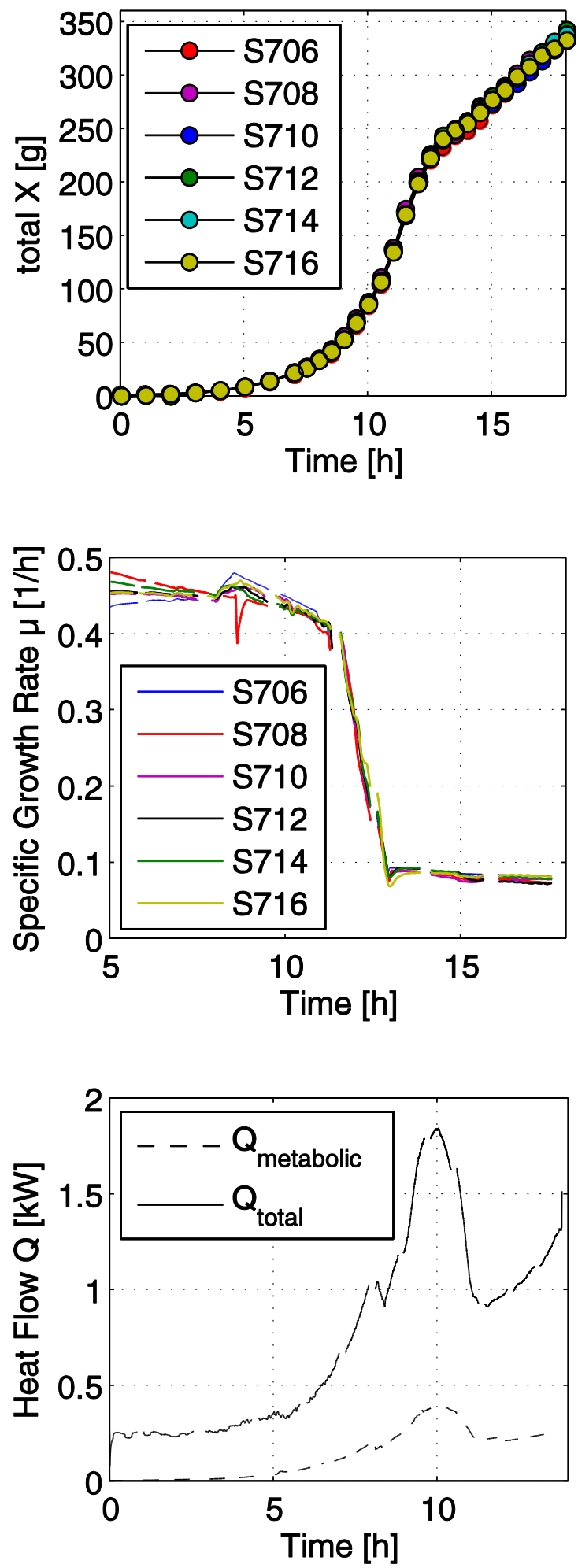

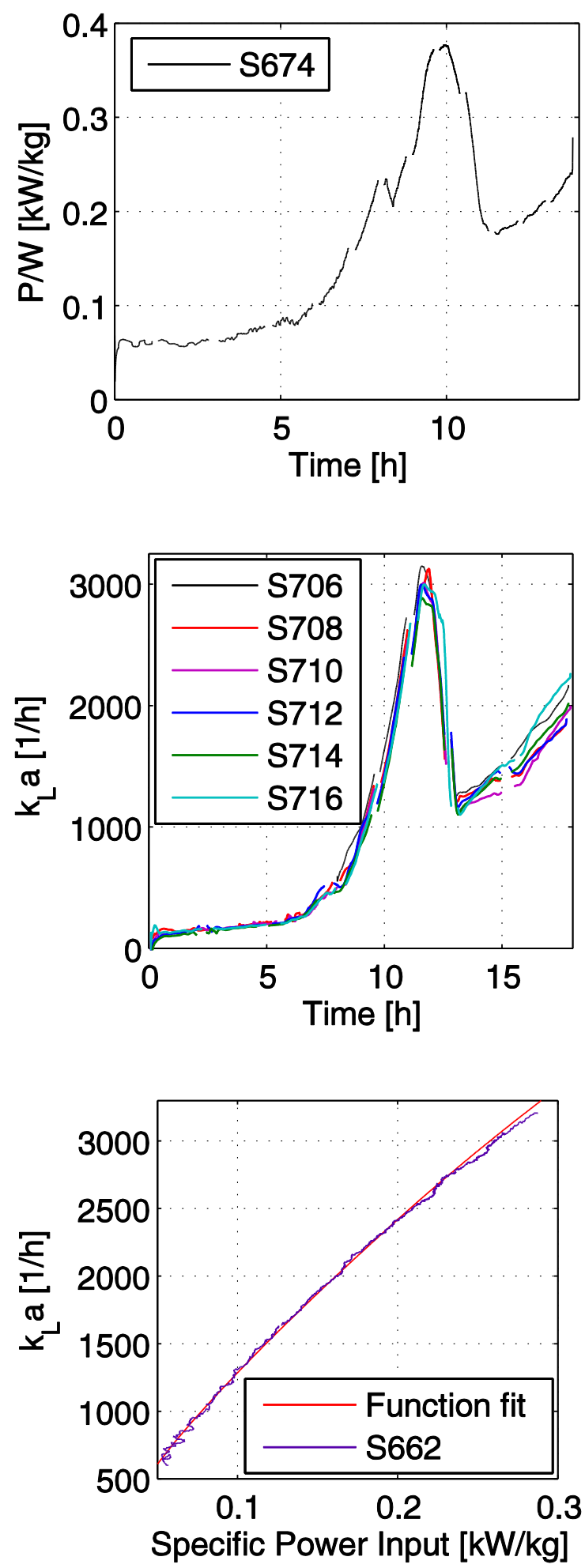